\title{
ESTRÉS ACADÉMICO Y TRABAJO DE GRADO EN LICENCIATURA EN EDUCACIÓN
}

\author{
Julio J. Aldana Z1* \\ https://orcid.org/0000-0002-7934-9103 \\ Josia Isea** \\ https://orcid.org/0000-0001-8921-6446 \\ Félix J. Colina Y2*** \\ https://orcid.org/0000-0002-6651-3509
}

RECIBIDO: Julio 2019 / ACEPTADO: Octubre 2019 / PUBLICADO: Enero 2020

Como citar: Aldana, Julio; Isea, Josia; Colina, Félix. (2020). Estrés Académico y Trabajo de Grado en Licenciatura en Educación. Telos: revista de Estudios Interdisciplinarios en Ciencias Sociales, 22 (1), Venezuela. (Pp. 91-105).

DOI: www.doi.org/10.36390/telos221.07

\section{RESUMEN}

La investigación tuvo por objetivo determinar el impacto del estrés académico durante el proceso de realización del trabajo especial de grado en estudiantes de Licenciatura en Educación. El estrés académico fue sustentado teóricamente desde la vertiente de autores como: Berrío y Mazo (2011), Castillo Pimienta et al. (2016), Méndez y Gálvez (2018), Moran et al. (2018). Metodológicamente, se trabajó con una investigación correlacional. La muestra de estudio, estuvo conformada por 167 estudiantes. La correlación de Pearson posee un resultado de 0,061, la cual se encuentra en el rango de correlación positiva muy débil y una significancia de 0,434, acercándose a 0,5 , generando un nivel de confianza positiva. Desde los resultados obtenidos se rechaza la hipótesis nula $\mathrm{HO}$. Se encontró una asociación lineal estadísticamente significativa, moderada ( $\mathrm{r}=-0.434, \mathrm{p}<0.05)$, entre el estrés académico y trabajo especial de grado, con lo cual, el estrés académico influye directamente en los estudiantes durante el proceso de

\footnotetext{
* Universidad Nacional Experimental Francisco de Miranda (UNEFM), Falcón, Venezuela. Doctor en educación. Magister en orientación en educación para padres. Licenciado en filosofia. Trabajando en el área de formación en investigación. Área de Psicología y orientación educativa. Docente investigador con la categoría de Asociado. Estudios Postdoctorales Estado, Políticas Públicas y Paz Social. Estudios Postdoctorales Integración y Desarrollo de América Latina. Desarrollados en URBE. Coordinador de la red de investigación Koinonía.

Autor corresponsal: julioaldanazavala@gmail.com

** Universidad Nacional Experimental Francisco de Miranda (UNEFM), Falcón, Venezuela. Doctor en Ciencias para el Desarrollo Estratégico. Maestría en Gerencia de Recursos Humanos. Licenciado en Educación, mención Filosofía. Trabajando en el área de formación en investigación. Área de Psicología y orientación educativa. Docente investigador con la categoría de Asociado. Estudios Postdoctorales Estado, Políticas Públicas y Paz Social. Estudios Postdoctorales Integración y Desarrollo de América Latina, desarrollados en URBE. Presidente de la fundación Koinonía. Correo electrónico: josiaisea@gmail.com

${ }^{* * *}$ Actualmente docente investigador en la Universidad Científica del Sur y Universidad Tecnológica del Perú, Lima, Perú. Doctor en ciencias de la educación. Magister en orientación de la conducta. Licenciado en educación. Trabajando en el área de gestión del conocimiento. Área de Psicología y orientación educativa. Docente investigador con la categoría de Asociado en la UNEFM. Estudios Postdoctorales Estado, Políticas Públicas y Paz Social. Estudios Postdoctorales Integración y Desarrollo de América Latina. Desarrollados en URBE. Miembro de la red de investigación Koinonía. Lima, Perú. Correos electrónicos: felixcolina260@hotmail.com; C19271@utp.edu.pe
} 
realización de trabajo especial de grado. El impacto del estrés académico ha influido negativamente sobre la muestra poblacional abordada, requiriéndose esfuerzos extras por parte de los estudiantes para la conclusión efectiva de los trabajos de investigación y concluir su proceso de formación en la universidad, siendo menester profundizar en investigaciones que permitan explicar con mayor detenimiento las consecuencias del estrés académico en la personalidad de los estudiantes. Es necesario recobrar el papel que juega la universidad en el fomento de nuevos conocimientos provenientes de trabajos de investigación, siendo pertinente considerar la planificación centrada en la investigación como una figura primordial para la generación de un currículo donde se aborde la importancia de acompañar desde las múltiples complejidades de los estudiantes investigadores durante el proceso de investigación.

Palabras clave: Estrés mental, efectos fisiológicos, investigación pedagógica, educación universitaria, metodología.

\section{Academic Stress and Degree Work in Bachelor of Education}

\section{ABSTRACT}

The research had for objective determine the Impact of the Academic Stress during the Process of Realization of the Special Work of Degree in Students of Degree in Education. Academic stress was supported theoretically from the perspective of authors such as: Berrío y Mazo (2011), Castillo Pimienta et al. (2016), Méndez y Gálvez (2018), Moran et al. (2018). Methodologically, a correlational investigation was carried out. The study sample consisted of 167 students. The Pearson correlation has a result of 0.061 , which is in the very weak positive correlation range and a significance of 0.434 , approaching 0.5 , generating a positive confidence level. From the results obtained, the null hypothesis $\mathrm{HO}$ is rejected. We found a statistically significant, moderate linear association ( $r P=-0.434, p<0.05)$, between academic stress and special grade work, with which, academic stress directly influences students during the process of carrying out special work of degree. The impact of academic stress has negatively influenced the population sample addressed, requiring extra efforts on the part of the students for the effective conclusion of the research work and to complete their training process in the university, being necessary to deepen in investigations that allow to explain with more attention the consequences of academic stress on the personality of students. It is necessary to recover the role played by the university in the promotion of new knowledge from research work, it being pertinent to consider research-centered planning as a key figure for the generation of a curriculum that addresses the importance of accompanying from the multiple complexities of student researchers during the research process

Keywords: Mental stress, fatigue (physiology), educational research, University education, Methodology.

\section{Introducción}

El accionar globalizante y competitivo donde se ven inmersos los estudiantes universitarios en formación de la carrera de educación, genera desafíos constantes consigo mismos y sus 
compañeros en procura de alcanzar el mayor éxito, reconocimiento, posible, especialmente cuando se encuentran en el proceso de trabajo especial de grado con la finalidad de cumplir con el último requisito académico para la obtención del título universitario que le confiere como licenciado en educación con la mayor calificación y honor al mérito. Es de destacar que el proceso investigativo puede configurar perspectivas de incertidumbre y tensión en los investigadores noveles (estudiantes), por cuanto deben presentar y sustentar un trabajo investigativo donde se destaque el cumplimiento de objetivos planteados en conformidad con líneas 0 áreas de investigación.

El proceso de investigación, aunado al cumplimiento del resto de requisitos académicos, pueden fomentar tensión en los estudiantes, concatenado con el accionar globalizante y competitivo del mundo actual, donde las plazas de trabajo cada día son más reducidas, siendo necesario cumplir con estándares de calidad exigidos por las organizaciones para cumplir con perfiles necesarios para la contratación, visualizan en el horizonte del estudiante universitario, la posibilidad de preocuparse en exceso por cumplir con tales exigencias, con la finalidad de cumplir con los requerimientos exigidos para la obtención de la titulación universitaria, en este sentido, La Universidad Autónoma de Occidente (2019, p. 1), plantea que:

El Trabajo de Grado es un ejercicio de profundización desarrollado por el estudiante de pregrado como requisito para optar al título profesional que mediante la integración y aplicación teórica o teórico-práctica de conocimientos y habilidades 0 a través de la generación de nuevo conocimiento, busca fortalecer las distintas competencias adquiridas durante su proceso de formación y, así mismo, contribuir al análisis y solución creativa de una problemática relacionada con el objeto de estudio o campo de acción de su profesión.

El trabajo especial de grado requiere de una alta disposición por parte de sus autores para lograr un trabajo efectivo, acorde a las expectativas esperadas por un jurado calificador, así como la comunidad científica en general, aunado a que es el último requisito de los estudiantes para lograr el cumplimiento del pensum de estudios y optar por su titulación profesional, con lo cual se ven inmiscuidas a lo largo del proceso de investigación, una complejidad de factores que pueden incidir positiva o negativamente en la realización del mismo, en el actual caso de investigación, se tomará como factor de estudio, el estrés académico, esto con la finalidad de abordar su influencia en los estudiantes que se preparan para optar al título profesional en la carrera de educación.

Desde lo planteado, el conglomerado de tensiones, preocupaciones, incertidumbres, esfuerzos por ser el mejor en la clase e investigación, pueden ser factores generadores del estrés académico, definido por Berrío y Mazo (2011, p. 65), como: "una reacción de activación fisiológica, emocional, cognitiva y conductual ante estímulos y eventos académicos". La realización del trabajo especial de grado, se constituye en un estímulo que puede tener una reacción favorable o desfavorable según la percepción del estudiante, originando una tensión positiva o negativa, es decir que le permita motivarse para trabajar en la conjugación de una investigación exitosa o por el contrario paralizarse o bloquearse para la realización de la misma, en ambos casos ocurre tensión como repuesta al estímulo generado. Por otro lado, Castillo Pimienta et al., 2016, (p. 236), plantean que:

En el marco de los actuales procesos de discusión respecto de la formación de pregrado, resulta importante poder ajustar las exigencias académicas de un modo 
razonable a fin de disminuir la sensación de agobio y sobrecarga experimentada por los estudiantes.

Se resalta la presencia de agobio y sobrecarga en los estudiantes, siendo generadores de estrés académico, lo cual puede influir además en el modo de cómo se perciben los estudiantes así mismos, configurándose un autoconcepto que podría ser positivo o negativo, en este sentido, Méndez y Gálvez (2018, p. 142), comenta que "el autoconcepto académico como conjunto de creencias y percepciones que van a guiar el comportamiento de los estudiantes en el ámbito de la educación formal".

Según sea el autoconcepto formado por los estudiantes será la reacción en pro de afrontar con asertividad el proceso de trabajo especial de grado, logrando transcender los posibles inconvenientes generados o sucumbir ante los mismos, fomentándose el estrés académico acompañado de síntomas afectadores de la salud integral de la persona, como la ansiedad, Moran et al. (2018, p. 207), plantea que:

Se observó que los universitarios con mayores niveles de ansiedad social presentan también mayores dificultades para regular sus emociones y, por el contrario, quienes no padecen niveles disfuncionales de ansiedad social pueden realizar un uso más adaptativo de sus estrategias de regulación emocional.

La autorregulación de las emociones, es un factor primordial para generar una salud mental equilibrada y poder afrontar con eficacia las dificultades propias de un proceso de investigación académica, sino se maneja adecuadamente, las reacciones podrían ser adversas consigo mismo y con el entorno social inmediato al estudiante, fomentándose un clima no asertivo para el establecimiento de relaciones interpersonales sinérgicas, más aun, sí el trabajo especial de grado se realiza en coautoría, los estudiantes involucrados deben manejar positivamente el estrés académico con la finalidad de articular un posicionamiento efectivo en la consecución de cumplir con los objetivos planteados tanto en la investigación como por los estudiantes investigadores de lograr con éxito su grado académico. En complemento, Aguayo Contreras y López Concha (2016, p. 2), aportan que:

Los distintos aspectos del Estrés Académico se relacionan con la Percepción de control del tiempo académico, pues se observan diferencias en el nivel, los estresores, las reacciones y las estrategias de afrontamiento de estudiantes que perciben controlar su tiempo de estudio con aquellos que no.

La percepción y auto regulación de las emociones son indispensables para que los estudiantes se fijen un autoconcepto apegado a una persona exitosa o vencida, en conformidad de afrontar asertivamente el proceso de trabajo especial de grado, siendo que sí el estrés académico es controlado y no logra transcender, los estudiantes podrán prevenir la aparición de síntomas contrarios al bienestar integral, como dolores de cabeza, fatiga, somnolencia, entre otros, prosiguiendo con lo planteado, Maceo, et. al. (2013, p. 193), destacan en su investigación que "las principales consecuencias del estrés fueron, la falta de concentración, fatiga intelectual, inapetencia a las tareas habituales, descuido, fatiga sexual, tristeza, descenso del rendimiento intelectual o físico, nerviosismo, modificación de la frecuencia cardiaca, tensión arterial, metabolismo y actividad física", lo cual puede incidir en el rendimiento académico, así como en el desarrollo efectivo del trabajo especial de grado.

En complemento de lo descrito, Toribio-Ferrer y Franco-Bárcenas (2016, p. 16), indican que "los principales estresores del estrés académico que los estudiantes presentan son: los exámenes, 
la sobrecarga de tareas y la personalidad y el carácter del profesor", contextualizando al trabajo especial de grado, se destaca la selección de tema de investigación, así como el asesor, tutor 0 director de trabajo especial de grado, siendo de los principales estresores en los investigadores noveles, aunado a la revisión documental, diseño de instrumentos, trabajo de campo, procesamiento estadístico o cualitativo de la data recopilada, construcción del informe escrito, cumplimiento de las normas metodológicas de la institución, financiamiento de los gastos derivados de la realización del trabajo especial de grado, son algunos elementos que pueden influir en la generación de estrés académico.

Prosiguiendo lo expuesto, en la Universidad Nacional Experimental Francisco de Miranda (UNEFM), como casa de estudio de educación superior y formadora de profesionales de la educación, requiere que sus estudiantes asuman un proceso de adaptación socio académico, para obtener una preparación durante diez semestres académicos que les permita formarse y ejercer competencias profesionales en un campo laboral, realizar prestación de servicio comunitario de forma obligatoria a la comunidad durante una duración de 120 horas académicas, así como la presentación oral de un trabajo especial de grado, siendo este el último eslabón para concluir con los requisitos para la obtención del grado académico de licenciatura en educación. Por consiguiente, se realizó un proceso de observación con los estudiantes del último semestre, con la finalidad de conocer a priori sus reacciones al estímulo que comprende el proceso de trabajo especial de grado desde su inicio hasta su finalización, se realizaron entrevistas no formales con el fin de conocer las causas y consecuencias que el estrés puede ocasionar.

Se observó que los estudiantes presentan un grado de irritabilidad, por cuanto se ven en la ansiedad de cumplir con todas las cargas que demanda ser estudiantes de último semestre, donde llevan consigo la preparación del trabajo especial de grado, de tal manera que la demasía de estudio provoca un cansancio que a su vez desencadena una serie de problemáticas como falta de concentración, debilidad, torpeza, lentitud, incapacidad para concentrarse, memorizar un simple párrafo $o$ un bloqueo mental al momento de desarrollar una actividad a causa de un miedo por no haberse preparado lo suficiente, ocasionándole inseguridad en su desempeño académico.

Lo cual puede originar que el trabajo especial de grado, se perciba como negativo, inalcanzable, no necesario para la formación docente, mientras que otros estudiantes conciben este proceso como una oportunidad para crecer y aprender sobre investigación, con la finalidad de formarse para ser docentes en aprendizaje permanente, siendo necesario investigar el impacto del estrés académico durante el proceso de realización del trabajo especial de grado en estudiantes de licenciatura en educación de la UNEFM, con la finalidad de conocer cómo se gestiona el estrés académico en los estudiantes durante el proceso de realización del trabajo especial de grado, con lo cual se podrán alertar medidas que configuren la posibilidad de gestionar estrategias académicas en pro de la salud integral de los estudiantes en conformidad de contar con futuros profesionales de la educación en condiciones óptimas de brindar apoyo en el crecimiento de la sociedad.

Considerando lo planteado, la presente investigación expone la siguiente pregunta: ¿Cuál es el impacto del estrés académico durante el proceso de realización del trabajo especial de grado en estudiantes de licenciatura en educación? Con el objetivo de determinar el impacto del estrés académico durante el proceso de realización del trabajo especial de grado en estudiantes de licenciatura en educación. 
A continuación, se presente el método y procesos metodológicos - estadísticos desarrollados durante la investigación:

\section{Método}

Se trabajó sobre la base de la investigación correlacional, Hernández, Fernández, Baptista (2014, p. 93), señalan que:

Tiene como finalidad conocer la relación o grado de asociación que exista entre dos o más conceptos, categorías 0 variables en un contexto en particular. Los estudios correlacionales, al evaluar el grado de asociación entre dos o más variables, miden cada una de ellas (presuntamente relacionadas) y, después, cuantifican y analizan la vinculación. Tales correlaciones se sustentan en hipótesis sometidas a prueba.

Al medirse de modo independiente las variables para luego generar la relación, la investigación se apoyó en investigación de tipo descriptiva, con un diseño no experimental, transeccional, de campo, por cuanto se realizó la medición de las variables de modo independiente, sin manipulación o experimentación de las mismas, registrándose los datos en una única recopilación por parte de los investigadores. Es de destacar que la correlación, de acuerdo a Hernández, et al., (2014, p. 94), puede ser positiva o negativa:

La correlación puede ser positiva o negativa. Si es positiva, significa que alumnos con valores altos en una variable tenderán también a mostrar valores elevados en la otra.

Si es negativa, significa que sujetos con valores elevados en una variable tenderán a mostrar valores bajos en la otra variable.

Esto implica que si es la correlación resulta positiva, el estrés académico puede influir en el proceso de realización del trabajo especial de grado, y este podría generar estrés académico en los estudiantes, en cambio sí resultare negativa, una de las dos variables se incrementaría proporcional sobre la otra, sin generar mayor incidencia. Así mismo, se analizó estadísticamente la correlación desde el coeficiente de correlación de Pearson, definido por Hernández, et al., (2014, p. 304), como "una prueba estadística para analizar la relación entre dos variables medidas en un nivel por intervalos o de razón. Se le conoce también como "coeficiente productomomento". Lo que permitió construir el sistema de hipótesis general:

Ha: El impacto del estrés académico se relaciona significativamente durante el proceso de realización del trabajo especial de grado en los estudiantes de licenciatura en educación.

H0: El impacto del estrés académico no se relaciona significativamente durante el proceso de realización del trabajo especial de grado en los estudiantes de licenciatura en educación.

La muestra de estudio, estuvo conformada por 167 estudiantes del décimo semestre de educación de la UNEFM de la ciudad de Coro, a quienes se les aplicó un instrumento de 31 ítems tipo escalamiento de Likert (cinco alternativas de repuestas: Extremadamente, Bastante, Moderadamente, Un poco, Nada), con la finalidad de medir las variables de estudio, el cual fue validado por el juicio de 5 expertos en el área de investigación, así como también obtuvo un grado de confiabilidad de 0,87 en el coeficiente Alfa de Cronbach, resultado obtenido de una prueba piloto aplicada a 20 individuos con características similares a la población de estudio. Se empleó el software SPSS 25 para el análisis de los datos estadísticos.

\section{Baremo para la correlación}

Para la ubicación del valor de correlación de Pearson, se tendrá en consideración el rango propuesto por Hernández, et al., (2010, p. 312):

Nivel de medición de las variables: intervalos o razón. 
Interpretación: el coeficiente $r$ de Pearson puede variar de -1.00 a +1.00 , donde: -1.00 = correlación negativa perfecta. ("A mayor $X$, menor $Y$ ", de manera proporcional. Es decir, cada vez que $X$ aumenta una unidad, $Y$ disminuye siempre una cantidad constante.)

Esto también se aplica "a menor $X$, mayor $Y$ ".

$-0.90=$ Correlación negativa muy fuerte.

$-0.75=$ Correlación negativa considerable.

$-0.50=$ Correlación negativa media .

$-0.25=$ Correlación negativa débil.

$-0.10=$ Correlación negativa muy débil.

$0.00=$ No existe correlación alguna entre las variables.

$+0.10=$ Correlación positiva muy débil.

$+0.25=$ Correlación positiva débil.

$+0.50=$ Correlación positiva media.

$+0.75=$ Correlación positiva considerable.

$+0.90=$ Correlación positiva muy fuerte.

+1.00 = Correlación positiva perfecta. ("A mayor $X$, mayor $Y$ " o "a menor $X$, menor $Y$ ", de manera proporcional. Cada vez que $\mathrm{X}$ aumenta, $\mathrm{Y}$ aumenta siempre una cantidad constante).

\section{Resultados}

\section{Estadística descriptiva}

Los resultados se focalizaron en describir tres áreas del estrés, con la finalidad de conocer su comportamiento en la población objeto de estudio y así, generar una clasificación de cual fase del estrés académico se encuentran:

\section{Área manifestaciones físicas. Conformada por los indicadores:}

Somnolencia, mayor necesidad de dormir. Cansancio y fatiga. Dolores de cabeza o jaquecas. Dolor de estómago y/o color irritable. Bruxismo (tensión de mandíbulas). Dolor de espalda. Taquicardia o palpitaciones fuertes. Aumento o disminución del apetito. Resfríos frecuentes (baja en el sistema inmune). Dificultades para dormir (insomnio o pesadillas).

Tabla 1 Área manifestaciones físicas

\begin{tabular}{lll}
\hline Alternativas & Frecuencia & $\%$ \\
\hline Extremadamente & 42 & 25 \\
Bastante & 53 & 32 \\
Moderadamente & 21 & 13 \\
Un poco & 37 & 22 \\
Nada & 14 & 8 \\
\hline
\end{tabular}

Fuente: elaboración propia.

Nota: La tabla representa los estadísticos descriptivos el área manifestaciones físicas.

La población de estudio se disgregó un $25 \%$ en la alternativa extremadamente; un $32 \%$ en la alternativa bastante; un $13 \%$ en la alternativa moderadamente, un $22 \%$ en la alternativa un poco y un $8 \%$ en la alternativa nada. Agrupando las alternativas enfocadas en evaluar el grado de estrés académico, se encuentra un $70 \%$ de la población en contraposición de un $30 \%$ que se encuentra en las alternativas un poco o nada, las cuales representan la percepción de los individuos de no padecer estrés académico. 
Área manifestaciones psicológicas y emocionales. Conformada por los indicadores:

Inquietud e hiperactividad. Tristeza y/o desgano. Ansiedad constante. Dificultades para concentrarse. Dificultades para pensar (quedarse en blanco). Problemas de memoria, olvidos frecuentes. Irritabilidad frecuente. Temor a no poder cumplir con las obligaciones. Baja motivación para actividades académicas. Angustia y/o ganas de llorar frecuente.

Tabla 2 Área manifestaciones psicológicas y emocionales

\begin{tabular}{lll}
\hline Alternativas & Frecuencia & $\%$ \\
\hline Extremadamente & 51 & 31 \\
Bastante & 27 & 16 \\
Moderadamente & 39 & 23 \\
Un poco & 35 & 21 \\
Nada & 15 & 9 \\
\hline
\end{tabular}

Fuente: elaboración propia.

Nota: La tabla representa los estadísticos descriptivos del área manifestaciones psicológicas y emocionales.

La población de estudio se disgregó un $31 \%$ en la alternativa extremadamente; un $16 \%$ en la alternativa bastante; un $23 \%$ en la alternativa moderadamente, un $21 \%$ en la alternativa un poco y un $9 \%$ en la alternativa nada. Agrupando las alternativas enfocadas en evaluar el grado de estrés académico, se encuentra un $70 \%$ de la población en contraposición de un $30 \%$ que se encuentra en las alternativas un poco o nada, las cuales representan la percepción de los individuos de no padecer estrés académico.

\section{Área manifestaciones conductuales. Conformada por los indicadores:}

Recuente tendencia a discutir con los demás. Necesidad de estar solo, aislarse. Uso de fármacos para estar despierto (auto medicados). Preferir otras actividades por sobre el estudio. Uso de fármacos para dormir (auto medicados). Dificultad para sentarse a estudiar ("sacar la vuelta"). Aumento del consumo de café y/o tabaco. Dejar de asistir a clases. Frecuente participación en carretes poco saludables. Tendencia a dejar de hacer, por desgano 0 apatía, lo que se acostumbra normalmente.

Tabla 3 Área manifestaciones conductuales

\begin{tabular}{lll}
\hline Alternativas & Frecuencia & $\%$ \\
\hline Extremadamente & 49 & 29 \\
Bastante & 58 & 35 \\
Moderadamente & 34 & 20 \\
Un poco & 14 & 9 \\
Nada & 12 & 7 \\
\hline
\end{tabular}

Fuente: elaboración propia.

Nota: La tabla representa los estadísticos descriptivos el área manifestaciones conductuales. La población de estudio se disgregó, un $29 \%$ en la alternativa extremadamente; un $35 \%$ en la alternativa bastante; un $20 \%$ en la alternativa moderadamente, un $9 \%$ en la alternativa un poco y un $7 \%$ en la alternativa nada. Agrupando las alternativas enfocadas en evaluar el grado de estrés académico, se encuentra un $70 \%$ de la población en contraposición de un $30 \%$ que se encuentra en las alternativas un poco o nada, las cuales representan la percepción de los individuos de no padecer estrés académico. 
Tabla 4 Resumen porcentual del estrés académico

\begin{tabular}{lll}
\hline Áreas & Padece estrés & No padece estrés \\
\hline Área manifestaciones físicas & $70 \%$ & $30 \%$ \\
Área manifestaciones psicológicas y emocionales & $70 \%$ & $30 \%$ \\
Área manifestaciones conductuales & $84 \%$ & $16 \%$ \\
Total Estrés Académico & $75 \%$ & $25 \%$ \\
\hline
\end{tabular}

Fuente: elaboración propia.

Nota: El $75 \%$ padece estrés académico en contraposición del $25 \%$ que no lo padece.

El promedio de resultados de un $75 \%$ de las áreas evaluadas, evidencia la presencia del estrés académico en estudiantes en proceso de realización y presentación del trabajo especial de grado, mientras que un $25 \%$ restante considera no padecer de estrés académico, generándose una proporción significante de población estudiada con la percepción de padecer estrés académico, siendo esto perjudicial para el sano equilibrio emocional, afectivo, conductual, de los individuos objeto de estudio.

Tabla 5 Dificultad para realizar el trabajo especial de grado

\begin{tabular}{lll} 
Elementos del trabajo especial de grado & fr & $\%$ \\
\hline Capítulo I (problema o contexto inicial) & 35 & 21 \\
Capitulo II (Referencial teórico) & 37 & 22 \\
Capitulo III (Referencial metodológico) & 46 & 27 \\
Capitulo IV (Resultados o teorización) & 31 & 19 \\
Capítulo V (Conclusiones o reflexiones finales) & 18 & 11 \\
\end{tabular}

Fuente: elaboración propia.

Nota: La tabla representa los estadísticos descriptivos sobre la dificultad para realizar el trabajo especial de grado por parte de los estudiantes encuestados.

La población de estudio se disgregó, un $21 \%$ para la opción capítulo I (problema o contexto inicial), $22 \%$ para la opción capitulo II (Referencial teórico), 27\% para la opción capitulo III (Referencial metodológico), 19\% para la opción capitulo IV (Resultados o teorización), mientras que la opción capítulo $\mathrm{V}$ (Conclusiones o reflexiones finales), obtuvo un $11 \%$, lo cual denota que los estudiantes le brindan mayor grado de dificultad a los primeros capítulos, por lo que el estrés académico se hace presente a lo largo de la realización del trabajo especial de grado. 
Tabla 6 Correlación de estrés académico y trabajo especial de grado

\begin{tabular}{llll} 
& & Estrés A. & Trabajo E. G. \\
\hline Estrés A. & Correlación de Pearson & 1 & 0,061 \\
& Sig. (bilateral) & & 0,434 \\
& $\mathrm{~N}$ & 167 & 167 \\
Trabajo E. G. & Correlación de Pearson & 0,061 & 1 \\
& Sig. (bilateral) & 0,434 & \\
& $\mathrm{~N}$ & 167 & 167 \\
\hline
\end{tabular}

Fuente: elaboración propia.

Nota: La tabla representa la correlación de Pearson entre estrés académico y trabajo especial de grado.

La correlación de Pearson posee un resultado de 0,061 , la cual se encuentra en el rango de correlación positiva muy débil y una significancia de 0,434 , acercándose a 0,5 , generando un nivel de confianza positiva. Desde los resultados obtenidos se rechaza la hipótesis nula $\mathrm{HO}$ : El impacto del estrés académico no se relaciona durante el proceso de realización del trabajo especial de grado en los estudiantes de Licenciatura en Educación. Aceptándose la hipótesis positiva Ha: El impacto del estrés académico se relaciona durante el proceso de realización del trabajo especial de grado en los estudiantes de Licenciatura en Educación.

Se encontró una asociación lineal estadísticamente significativa, moderada ( $\mathrm{rP}=-0.434, \mathrm{p}<0.05)$, entre el estrés académico y trabajo especial de grado, con lo cual, el estrés académico influye directamente en los estudiantes durante el proceso de realización de trabajo especial de grado.

\section{Discusión}

El objetivo de esta investigación fue determinar el impacto del estrés académico durante el proceso de realización del trabajo especial de grado en estudiantes de Licenciatura en Educación. Los resultados evidencian que el estrés académico tiene un impacto del $75 \%$ en la población encuestada, traducido a un coeficiente de Pearson de 0,061, la cual se encuentra en el rango de correlación positiva muy débil, en función de lo planteado por Hernández, Fernández, Baptista (2010, p. 312), con lo cual se declara que existe impacto del estrés académico en los estudiantes, viéndose influenciado la realización del trabajo especial de grado, de modo negativo, por cuanto los estudiantes pueden sufrir traumas emocionales, afectivos, conductuales, a lo largo del proceso, viéndose afectada su salud mental y física, siendo esto además un indicador que afecta la sana convivencia de los estudiantes consigo mismos y su entorno social.

Los resultados concuerdan con lo planteado por Aiquipa, Ramos, Curay \& Guizado (2018), plantean que existen diversos factores que influyen en la realización del trabajo especial de grado, entre los cuales se encuentran relacionados al aspecto psicológico y estrés, siendo pertinente que las universidades consideren la variable socio emocional como factor determinante en la producción científica por parte de los estudiantes, situación que amerita enfocar el currículo desde una vertiente flexible, dinámica, con la finalidad de generar la incorporación de estrategias en apoyo a superar la problemática planteada, lo cual se dificulta 
cuando se siguen procesos educativos verticales desde una concepción tradicional de transmisión de contenidos, lo cual se distancia del enfoque centrado en el estudiante como protagonista del aprendizaje, mediante la investigación como medio para la generación de conocimientos en contexto de aprender a lo largo de la vida.

En complemento, lanni Gómez (2017) señala la importancia del docente como orientador de los procesos académicos, involucrando el acompañamiento motivacional hacia los estudiantes con la finalidad de contribuir en la generación de aprendizajes significativos basados desde la investigación, así mismo, Ochoa Sierra \& Cueva Lobelle (2017, p. 85), destacan que:

La motivación del director (tutor) también es crucial: un director al que le interese el tema del estudiante lo apoyará de forma decidida y contribuirá al éxito de la investigación, de la tesis y de la publicación de los resultados.

No puede dejarse a merced del mero esfuerzo realizado por los estudiantes en la realización del trabajo especial de grado, se hace necesaria la participación cooperativa de los actores educativos que hacen vida en la institución para promover no solo la realización de investigaciones efectivas, sino, que sean generadas en un marco de interacción donde se promuevan las competencias emocionales pertinentes para minimizar el estrés y sus impactos negativos en la persona.

\section{Conclusiones}

El impacto del estrés académico ha sido bajo a moderado, sin embargo puede influir negativamente sobre la muestra poblacional abordada, requiriéndose esfuerzos extras por parte de los estudiantes para la conclusión efectiva de los trabajos de investigación y concluir su proceso de formación en la universidad, siendo menester profundizar en investigaciones que permitan explicar con mayor detenimiento las consecuencias del estrés académico en la personalidad de los estudiantes, así como que otros factores pueden generar o profundizar el mismo.

La realización del trabajo especial de grado, requiere de gran atención y concentración por parte de los investigadores para lograr su óptima realización, debiendo trabajar diversas competencias para articular la efectividad del trabajo investigativo, siendo una de ellas la competencia lingüística, en este sentido, Escudero-Sepúlveda, Escudero-Sepúlveda y Rodríguez-Morales (2012, p. 1), comentan que:

Concordamos en que un manuscrito debe escribirse correctamente, con buen dominio del lenguaje y de la ortografía. En pregrado, donde los estudiantes tienen la posibilidad de comenzar a investigar y que esto se haga parte de su proceso de formación científica.

Generar la concentración pertinente es sinónimo de conciliar una redacción investigativa acorde a las exigencias de calidad que requiere una investigación para ser presentada ante un jurado calificador, así como ser colgada en un repositorio institucional donde será leída por un incógnito lector, siendo necesaria por parte de los investigadores contar con la salud mental acorde para efectuar esta función, siendo menoscaba por la presencia del estrés académico, el cual puede generar la realización de una redacción académica no acorde a las expectativas esperadas. Así mismo, Mayz y Pérez (2002, p. 1), disertan al plantear que:

Existen suficientes razones para justificar la investigación científica en las universidades. Ésta debe ser la base de la educación, pues enseña a pensar, a 
comprender y a crear nueva información, conocimientos y tecnologías. Permite, además, formar profesionales con creatividad, dispuestos a innovar, curiosos. Es importante resaltar que el conocimiento, la información y las tecnologías generados a través de la investigación juegan un gran papel en el desarrollo integral del país; por lo tanto, debería existir una mayor articulación entre los organismos de ciencia y tecnología y las universidades.

La generación de nuevos conocimientos desde las universidades es esencia de las mismas, transcendiendo así la mera enseñanza mecanicista, la investigación desde lo abordado permite el fomento de un futuro profesional en capacidad de ser proactivo, reflexivo, indagador, propiciador de nuevas alternativas en su área de trabajo, por lo cual, la investigación es un eje primordial en el proceso de aprendizaje, con lo cual se les debe brindar a los estudiantes de pregrado en formación investigativa, el acompañamiento y el apoyo logístico pertinente para configurar un trabajo de investigación donde el estrés académico no sea un protagonista central. Complementando lo planteado, la investigación es fundamental para concebir una universidad en plenitud de sus funciones académicas, Rodríguez Abrahantes, Rodríguez Abrahantes y García Pérez (2016, p. 1), al referirse a la investigación, señalan que "esta constituye un eje integrador, central en el proceso de formación profesional para la adquisición de conocimientos, competencias y aptitudes en los graduados".

Las universidades no solo deben concebir el trabajo de investigación como un requisito de graduación, sino, como una estrategia para la formación de competencias en los estudiantes que les permitan desempeñarse en la vida social y laboral de un modo asertivo, es decir, en consonancia con las expectativas de competitividad de la sociedad del conocimiento y de la tecnología, esto permitiría repensar los procesos de investigación e incorporar alternativas donde se tenga en consideración la presencia del estrés académico durante el proceso de realización del trabajo especial de grado, siendo posible establecer medidas preventivas para la minimización o erradicación del estrés académico en los estudiantes inmersos en proceso de investigación.

Es de destacar que parte del estrés académico puede influir negativamente en el estado de ánimo o motivación de los estudiantes que realizan el trabajo especial de grado, en este sentido, Gutiérrez \& Mayta (2003, p. 1), contribuye exponiendo que:

El entusiasmo del estudiante para investigar se hace evidente en un principio con proyectos ambiciosos e ideas innovadoras, pero este ánimo se va perdiendo con el tiempo; de los trabajos concluidos la mayoría son presentados como requisito en una asignatura (para luego ser archivados) y muy pocos llegan a ser publicados en revistas indexadas.

La motivación debe ser un factor central en los investigadores para realizar un trabajo de investigación acorde a las exigencias de la comunidad científica internacional, por cuanto es necesario investigar para el mundo global, así las universidades deben articular entre sus planes formativos, el acompañamiento y apoyo que permita a los investigadores mantener la motivación y no caer en la frustración, desánimos, desesperanzas aprendidas, que podrían influenciar en el estancamiento o imposibilidad de realización del trabajo investigativo, el estrés académico no puede convertirse en un factor propiciador del fracaso investigativo, en el mejor de los casos debería convertirse en un "eustrés", beneficiador de la motivación al logro para lograr el cumplimiento de metas personales y profesionales. 
Prosiguiendo con lo planteado hasta ahora sobre la transcendencia de la investigación como un mero acto o requisito académico, Calvo López, 2009, p. 39), apoya al afirmar:

Los tres casos han permitido mostrar que la titulación universitaria no puede considerarse como un mero trámite formal, sino como un proceso complejo en el que los estudiantes producen conocimiento, entendiendo por ello la movilidad de temas, conceptos y problemas que ellos hacen circular en la tesis a manera de red de significaciones, apropiándose de los significados y plasmándolos mediante el despliegue de recursos de escritura.

Es necesario recobrar el papel que juega la universidad en el fomento de nuevos conocimientos provenientes de trabajos de investigación, siendo pertinente considerar la planificación centrada en la investigación como una figura primordial para la generación de un currículo donde se aborde la importancia de acompañar desde las múltiples complejidades de los estudiantes investigadores durante el proceso de investigación, la minimización o control del estrés académico, esto con la finalidad de propiciar un clima de aprendizaje donde el estudiante investigue para crecer, teniendo la certeza y convicción que está contribuyendo a la edificación de la sociedad del conocimiento, siendo una posibilidad real para transcender el estado donde primordialmente se percibe el trabajo especial de grado como un mero requisito de graduación sin mayor importancia que esa, con lo cual, los estudiantes podrían verse influenciados negativamente hacia un proceso de desmotivación.

Siendo necesario fomentar en los estudiantes que se encuentran en proceso de trabajo especial de grado, una actitud positiva, Barrientos Oradini, et. al. (2019, p. 175), plantean que "la visión positiva hacia el futuro significa la expectativa de obtener resultados favorables en la vida de los individuos, mostrando autoestima y generando vías de logro de objetivos más exitosas al tomar mejores decisiones frente a contingencias presentadas", por cuanto una percepción positiva de sí mismo contribuye a transcender las múltiples problemáticas y factores estresores durante la realización del trabajo especial de grado.

\section{Referencias Bibliográficas}

Aguayo Contreras, Liz, y López Concha, Camila (2016). Estrés académico en estudiantes de cuarto año de educación diferencial de la universidad de Concepción. Tesis de grado. Licenciado en educación. Universidad de Concepción, Chile. Recuperado de http://repositorio.udec.cl/bitstream/handle/11594/2996/Tesis_Estres_Academico_en_ estudiante_de_cuarto_a\%C3\%B10_de_educacion_diferencial.Image.Marked.pdf?seq uence=1\&isAllowed =y Consulta: $23 / 06 / 19$

Aiquipa, Jesús J., Ramos, Cinthia M., Curay, Rosmery, \& Guizado, Leidy L. (2018). Factores implicados para realizar o no realizar tesis en estudiantes de psicología. Propósitos y Representaciones, 6(1), 21-82. https://dx.doi.org/10.20511/pyr2018.v6n1.180 Consulta: 15/10/19

Barrientos Oradini, Nicolás, Araya Castillo, Luis, Veloso Salazar, Victor, \& Herrera Ciudad, Claudia. (2019). Optimismo disposicional en docentes de universidades privadas en región metropolitana en Chile. Revista Arbitrada Interdisciplinaria Koinonía, 4(7), 168-184. doi:http://dx.doi.org/10.35381/r.k.v4i7.199 Consulta: 21/06/19

Berrío García, Nathaly., \& Mazo Zea, Rodrigo (2011). Estrés Académico. Revista de Psicología. Universidad de Antioquia, 3(2), 65-82. Recuperado de 
http://pepsic.bvsalud.org/scielo.php?script=sci_arttext\&pid=\$2145-

48922011000200006\&lng=pt\&tlng=es Consulta: 22/05/19

Calvo López, Mónica (2009). La elaboración de la tesis de licenciatura como espacio para la formación y la construcción social del conocimiento. Perfiles educativos, 31(124), 2241. Recuperado

http://www.scielo.org.mx/scielo.php?script=sci_arttext\&pid=S018526982009000200003\&lng=es\&tlng=es Consulta: 18/04/19

Castillo Pimienta, Carlos, Chacón de la Cruz, Tomas, \& Díaz-Véliz, Gabriela (2016). Ansiedad y fuentes de estrés académico en estudiantes de carreras de la salud. Investigación en educación médica, 5(20), 230-237. https://dx.doi.org/10.1016/j.riem.2016.03.001 Consulta: 26/05/19

Escudero-Sepúlveda, Andrés, Escudero-Sepúlveda, Julián, \& Rodríguez-Morales, Alfonso. (2012). La redacción de un trabajo científico. Investigación Clínica, 53(1), 111-112. Recuperado de http://ve.scielo.org/scielo.php?script=sci_arttext\&pid=S053551332012000100010\&lng=es\&tlng=es Consulta: 23/06/19

Gutiérrez, César \& Mayta, Percy (2003). Publicación desde el Pre Grado en Latinoamérica: Importancia, Limitación y Alternativas de Solución. CIMEL. 8(1): 54-60. Recuperado de http://sisbib.unmsm.edu.pe/BVRevistas/cimel/v08 n1/PDF/a10v8n1.pdf Consulta: 23/06/19

Hernández, Roberto, Fernández, Carlos, Baptista, Pilar (2010). Metodología de la Investigación (quinta edición). Editorial Mc - Graw - Hill Interamericana. México.

Hernández, Roberto, Fernández, Carlos, Baptista, Pilar (2014). Metodología de la Investigación (sexta edición). Editorial Mc - Graw - Hill Interamericana. México.

Lanni Gómez, Carol Elizabeth (2017). MIRAMDA: Una propuesta educativa emergente desde la investigación. Revista Arbitrada Interdisciplinaria Koinonía, 2(3), 9-30. Recuperado de http://fundacionkoinonia.com.ve/ojs/index.php/revistakoinonia/article/view/49/36 Consulta: $15 / 10 / 19$

Maceo Palacio, Omar, Maceo Palacio, Alberto, Varón Blanco, Yalili, Maceo Palacio, Maricel, \& Peralta Roblejo, Yoandris (2013). Estrés académico: causas y consecuencias. MULTIMED,

Recuperado

de

http://www.revmultimed.sld.cu/index.php/mtm/article/view/302/410 Consulta: 20/06/19 Mayz, Juliana, \& Pérez, Julio (2002) ¿para qué hacer investigación científica en las universidades venezolanas? Investigación y Postgrado, 17(1), 159-171. Recuperado de http://ve.scielo.org/scielo.php?script=sci_arttext\&pid=S131600872002000100007\&lng=es\&tlng=es Consulta: 23/06/19

Méndez, Juan, \& Gálvez, José (2018). Propiedades psicométricas de la Escala de Autoconcepto Académico (EAA) en estudiantes universitarios chilenos. Liberabit, 24(1), 131-145. https://doi.org/10.24265/liberabit.2018.v24n1.09 Consulta: 23/06/19

Moran, Valeria, Olaz, Fabian, Pérez, Edgardo, \& Del Prette, Zilda (2018). Desarrollo y validación del Test de Ansiedad Social para estudiantes universitarios (TAS-U). Liberabit, 24(2), 195-212. https://doi.org/10.24265/liberabit.2018.v24n2.03 Consulta: 23/06/19

Ochoa Sierra, Ligia \& Cueva Lobelle, Alberto (2017). El bloqueo en el proceso de elaboración de una tesis de maestría: angustias y desazones percibidas por sus protagonistas. 
Recuperado de http://www.scielo.org.co/pdf/leng/v45n1/0120-3479-leng-45-0100061.pdf Consulta: 15/10/19

Rodríguez Abrahantes, Taimi, Rodríguez Abrahantes, Arahy, \& García Pérez, Margarita (2016). La investigación y su contribución formativa en estudiantes de las ciencias médicas. EDUMECENTRO, $8(1)$, 143-158. Recuperado de

http://scielo.sld.cu/scielo.php?script=sci_arttext\&pid=S2077-

28742016000100011\&lng=es\&tlng=es Consulta: 19/06/19

Toribio-Ferrer, Claudia, \& Franco-Bárcenas, Silvia (2016). Estrés Académico: El Enemigo Silencioso del Estudiante. Salud y Administración, Volumen 3 Número 7 Enero-Abril 2016.

pp. 11 18. Recuperado

de

http://www.unsis.edu.mx/revista/doc/vol3num7/A2_Estres_acad.pdf Consulta: 23/06/19

Universidad Autónoma de Occidente (2019). Trabajo de grado. Recuperado de https://www.uao.edu.co/informacion/trabajo-de-grado-pregrado-general. Consulta: 23/06/19 